\title{
Low Temperature Research: Methods and Results
}

\author{
By Prof. F. A. Lindemann, F.R.s., Professor of Experimental Philosophy, \\ University of Oxford
}

$\mathrm{F}^{\mathrm{EN}}$ W branches of scientific research have developed more rapidly in the course of the last quarter of a century than the investigation of the properties of matter at low temperatures. Twenty-five years ago such problems seemed of comparatively slight fundamental interest. One knew, of course, that the electrical conductivity of metals increased with falling temperature. Dewar had shown that the specific heats of solids diminished somewhat. But no striking new phenomena were expected, and no exciting theoretical developments appeared likely to arise from research in these regions. Low-temperature work had gone out of fashion. In Leyden alone, in the magnificently equipped laboratory of Kamerlingh Onnes, a series of elaborate and most accurate measurements of all sorts of properties of a series of substances attested that interest in this branch of knowledge was not extinct.

As so often happens, it was from quite an unexpected quarter that interest in the subject was revivified. In 1905 Nernst had enunciated his famous third law of thermodynamics, according to which, in any thermodynamic process, at the absolute zero the rate of change of affinity with temperature equals the rate of change of the heat content. To test this, the atomic heats near the absolute zero had to be ascertained, and to this end, with characteristic energy, Nernst turned over his Berlin laboratory to low temperature research. It very soon emerged, somewhat unexpectedly, that at low temperatures the atomic heats of all solids seemed to tend to zero. The kinetic implications of this discovery were farreaching.

Already in the year 1900 Planck had enunciated his famous law of complete radiation. In order to derive this, it had been necessary to assume that linear oscillators obeying Maxwell's equations could only absorb or emit radiation in quanta the energy of which was proportional to their frequency. But since it followed from Kirchhoff's law that the same law of radiation must emerge whatever the radiating entities, nobody had felt constrained to attach great importance to Planck's revolutionary presupposition. True, linear oscillators on his premises would lead to a law agreeable to experiment, but then no one really believed that the radiation from a hot body was due to linear Hertzian oscillators. Thus Planck's epochmaking assumption was treated with the com- fortable indifference with which one slurs over the details of processes introduced in so many imaginary thermodynamic eycles.

Even when Einstein pointed out in 1907 that the variation with the temperature of the specific heat of the diamond could be accounted for by applying Planck's premise to the atoms, it aroused little interest. For the diamond had long been known to form an exception to Dulong and Petit's law, and one was accustomed to attribute the anomaly to polymerisation or some similar question-begging explanation.

When it appeared that not only the atomic heat of the diamond, but also that of all solids, diminished at low temperatures, the matter took on a new complexion; and after it had been shown that the atomic heats of all simple substances when plotted against the temperature could be represented by the same formula, containing only one parameter characteristic of the substance, it became clear that, far from being a special assumption introduced in an imaginary case-unimportant, since any permissible imaginary process must lead to the right result-Planck's premise was a very vital physical fact.

If actual atoms in a space-lattice held in position by their mutual attractions and repulsions could only take up or lose energy in quanta proportional to their frequency, then there must be something radically wrong with the whole basis of classical dynamics. The atomic heat measurements at low temperatures seemed to prove the major premise ; and though we have now learnt to express it in the form that there are only a finite number of distinguishable states within a given energy range for the atoms in a crystal, the minor premise has finally won general acceptance. Differences may still exist as to the best way of introducing the quantum hypothesis. Nobody would to-day deny that some definite break with the classical point of view was inevitable.

Until 1933 the methods used for producing low temperatures were the same in principle as were used by Dewar, Linde and Hampson, and indeed by Cailletet and Pictet. The obvious way to cool a substance is to place it in thermal contact with a substance colder than itself. Any substance will do, and it is clear that, in the first stages at any rate, a gas will be the easiest to cool; for to reduce the temperature we must slow up the motion of the molecules. There are two simple 
ways of doing this. The first is to let them collide with particles moving away from them. If a gas molecule is reflected from a fixed surface at the same temperature as the gas, its velocity on the average will be as great after the collision as before. But if the surface is not fixed but receding, the effective relative speed at the moment of collision will be reduced. Hence if gas molecules, instead of being enclosed in a vessel with fixed walls, are reflected from, say, a receding piston, their velocity will be lowered and the temperature of the gas will fall. This is what happens when the temperature is reduced by the conversion of heat into external work. The second method to cool a gas is to allow it to expand, that is, allow the molecules to recede from one another. If they naturally attract one another appreciably, this mutual attraction will tend to reduce their speed as they move apart, in other words, to reduce the temperature. This is the so-called Joule-Thomson effect which has been largely used in liquefying gases.

A liquid gas produced by either of these methods, or a combination of the two, can be further cooled by pumping off the gas above the liquid. This causes more molecules to escape than return, and thus by doing work against the mutual attraction, as in the Joule-Thomson effect, tends to cool the liquid. A limit is set to this method of cooling, of course, by the fact that the vapour pressure diminishes exponentially with falling temperature. When the number of molecules which evaporate in unit time is so small that their latent heat just balances the inflow of heat due to imperfect insulation, no further reduction of temperature can be achieved. By these means, using liquid helium and a battery of the most efficient pumps obtainable, a temperature of $0.7^{\circ}$ was reached at Leyden by Keesom.

A totally different method, however, originally suggested by Debye and Giauque, has brought quite a new temperature region within our reach. The entropy of a substance is a measure of its state of disorder. A set of molecules at rest in an accurate space-lattice would be in a state of complete order, that is, their entropy would be zero. As soon as their positions or motions vary, the entropy increases. Now paramagnetic salts contain atoms which behave like small magnets. Obviously, if their axes are all aligned parallel to one another, their positional state of order will be greater than when they are oriented at random. If we apply an external magnetic field, the axes of the magnetic atoms will all set themselves parallel to the lines of force. The entropy due to their positional disorder will therefore decrease; hence if the substance is thermally insulated, the entropy due to their thermal motion must increase ; in other words, heat will be developed. Now if this heat is carried away, for example, by placing the substance in contact with liquid helium, and the substance is again thermally insulated, it is clear that one can cool the substance by reversing the process. For when the external field is removed the axes of the atomic magnets will tend, under the influence of thermal agitation, to resume their natural disordered condition, the positional entropy will increase at the expense of the entropy of agitation and the temperature must fall.

This effect was utilised by Giauque in California and by de Haas and Wiersma in Leyden in 1933 and by Kürti and Simon in Oxford in 1934 to cool substances to extremely low temperatures. Experiments in the region between $0.03^{\circ}$ and $1^{\circ}$ can be carried out without difficulty, and at Leyden, where a very strong magnet is available, a temperature of $0.005^{\circ}$ has actually been reached. A great deal of work will have to be done before this method is fully exploited, as for each tem. perature range a suitable paramagnetic salt is required, but there is little doubt that by employing it in cascade or otherwise, extremely low temperatures will be as readily accessible to research as were temperatures a thousand times higher twenty-five years ago.

The measurement of these low temperatures naturally presents quite a new set of problems. Down to $1^{\circ}$, of course an ordinary helium gas thermometer can be used; further, since all its constants are known, one can calculate the vapour pressure of helium and utilise this as a measure of the temperature down to $0.7^{\circ}$ or even $0.5^{\circ}$. But at $0 \cdot 1^{\circ}$ the vapour pressure of helium is $10^{-30} \mathrm{~mm}$. and at $0.03^{\circ}$ it is $10^{-102} \mathrm{~mm}$. of mercury. Quite different methods must therefore be employed to measure such temperatures.

Fortunately, one such method is ready to hand. The magnetic susceptibility of a paramagnetic salt is a measure of the ease with whioh the magnetic atoms can be oriented. The smaller the thermal agitation the more readily will they be directed by an external field. Thus, roughly speaking, the susceptibility will be inversely proportional to the temperature. Obviously this rule is no more than a first approximation. Accurate thermometry will only be possible when the susceptibility curve has been linked up to the gas thermometer curve by means of proper thermodynamic cycles, a research now in hand at Oxford. Once this has been done, these susceptibility measurements will form a very convenient form of thermometry enabling an accuracy of $10^{-5}$ degrees or even better to be achieved.

Most of the phenomena at low temperatures have fallen into line with the predictions or at any rate the explanations of the quantum 
dynamics. The atomic heats of simple substances agree as well as can be expected with the theoretical expressions, especially when one remembers the difficulty of working out and weighting all the modes of oscillation of a space-lattice of atoms or even complicated molecules. The atomic frequencies calculated from elastic constants agree with those derived from the atomic heat curves; even the fine structure in the spectral lines due to nuclear spin is mirrored in anomalies in the specific heats at the calculated temperatures. Quantum considerations, as Simon showed, enable one to understand the curious fact that helium remains liquid, in the sense that it has a very low viscosity, down to the lowest temperatures, though there is a point at which a certain order tends to be established. For with liquid helium the zero point energy is so great that at atmospheric pressure the substance can never become solid. If the pressure is raised, it can be reduced to the crystalline state even at temperatures ten times as high as its boiling point.

There is, however, one phenomenon, and that a very striking one, that has so far defied adequate explanation. In 1913 Kamerlingh Onnes announced that when mercury was cooled below $4^{\circ}$ its electrical resistance vanished. Supra-conductivity, as this effect is called, was afterwards found to occur in lead, tin and a number of other metals, though not by any means in all, for example, not in copper as low as $0.05^{\circ}$. Even some semi-conductors exhibit the same property, such as niobium carbide-indeed in this substance supra-conduction sets in at the highest temperature so far observed, namely, $12^{\circ}$. All attempts to observe some trace of electrical resistance in the supra-conducting state have failed. A current induced in a ring of supra-conducting material continues to run with undiminished strength for days on end. The phenomenon does not seem susceptible of explanation by any of the ordinary theories of electron conduction.

Much work has been done in Leyden, Berlin, Toronto and Oxford on this strange effect, and the somewhat complicated phenomena are gradually being disentangled. But whether it will be possible to fit it into the general scheme or whether it may not require some new mode of approach it is too early yet to say.

Though more low temperature research has been carried out in the last twenty-five years than in all preceding periods put together, and though temperatures within one hundredth of a degree from the absolute zero have been attained, there is no question of our having reached finality.

Our nearness to the absolute zero is apparent rather than real; if we had chosen to measure the temperature on a geometric rather than an arithmetic scale, it would have been less convenient in many ways; but it would have made it clear that, towards low temperatures, as towards high, there is always an infinite distance ahead of us; and in each such range we may expect new effects and new phenomena.

Fortunately, there are within the Empire laboratories where these effects can be studied. At Oxford, Cambridge and Toronto, work at liquid helium temperatures and below is being done. It may be hoped that the next generation will see the position of pre-eminence enjoyed by England in Dewar's time recaptured, and that advances as valuable and important as those which have signalised the past quarter of a century await us in the next.

\section{Cosmic Rays}

By Dr. Arthur H. Compton, University of Chicago, and George Eastman Visiting Professor in the University of Oxford

\section{$\mathrm{T}$} HE twenty-five year period of King George's reign includes almost the entire history of the study of cosmic rays. The presence of these rays was revealed by a series of experiments carried on between 1909 and 1914. Wulf, on the Eiffel Tower, and Gockel, flying in a balloon to 4,500 metres, found that rays from radioactive sources in the ground could not account for the ionisation observed at high altitudes, and suspected some radioactive material in the upper atmosphere'. Hess, in a series of notable balloon flights, found an actual increase of ionisation with increasing altitude, and concluded "that a radia- tion of very high penetrating power enters our atmosphere from above". These experiments were at first criticised by other investigators, but were quickly confirmed by the more precise observations of Kolhörster, and have since been found correct in all their essentials.

After eleven quiescent years, Millikan made some bold speculations regarding the origin of these penetrating rays, which showed in a striking manner that their study might well give important new information regarding the evolution of the universe. Largely through his experiments and those of Hoffmann, the existence of the radiation 